\title{
Hybrid Optical Network Architectures: Bringing Packets and Circuits Together
}

\author{
Christoph M. Gauger and Paul J. Kühn, Universität Stuttgart \\ Erik Van Breusegem, Mario Pickavet, and Piet Demeester, Ghent University - IBBT - IMEC
}

\begin{abstract}
In recent years hybrid optical network architectures, which employ two or more network technologies simultaneously, were proposed. They aim at improving the overall network design by combining the advantages of different technologies while avoiding their disadvantages. In order to structure this developing research field, we classify such hybrid architectures based on the degree of interaction and integration of the network technologies. Also, we discuss the three classes and their main representatives regarding key characteristics, performance benefits, and realization complexity. Finally, we highlight two hybrid architectures and show their key benefits compared to the respective non-hybrid architectures through a dimensioning case study.
\end{abstract}

\section{INTRODUCTION}

In the early days of the Internet, network bandwidth was a scarce resource, certainly relative to the computing power available. Thus, the (original) design of the TCP/IP protocol suite mostly targeted networks that today would not qualify as broadband. The rapid development and deployment of fiber transmission technology, most notably wavelength-division multiplexing (WDM), shifted this balance. Suddenly, there was more transmission capacity available than even specialized routers could handle. Since then, electronics have been struggling to match the pure raw speeds of optics.

To relieve electronics from bulk processing and switching, optical concepts like wavelength, waveband, and even fiber switching were developed and are currently being introduced, such as reconfigurable optical add-drop multiplexers (ROADMs) and all-optical crossconnects (OXCs). In these networks optical devices and systems are the workhorses, and electronics introduce intelligence to the data and control plane.

However, this bulk transport in optics can also be very bandwidth inefficient, especially in networks with bursty traffic patterns. This is mostly because wavelength switching has a very coarse granularity and is rather slow. Its adaptation times range up to hours or higher, and are thus much larger than critical timescales of traf- fic dynamics. Many researchers hope that highly dynamic all-optical switching may diminish this inefficiency. Unfortunately, as all-optical switching and processing are not ready for deployment, this dream still has not become reality.

So the essential question how to design highly adaptive networks, when faced with an electronic bottleneck, still remains unanswered. A closer look at the major challenges of network efficiency, node scalability, and heterogeneous service requirements leads to fundamental questions on the characteristics of the design space:

- Will bandwidth become so cheap that inefficiencies of bulk (wavelength) switching become irrelevant? Probably not. So what do we do to increase efficiency?

- Agile optical systems still have limited functionality and their mostly analog nature indicate that their realization complexity will remain high for some years to come. So, how can we minimize node complexity and thus ensure scalability?

- How do we support the increasingly heterogeneous service requirements regarding bandwidth and quality of service? Some applications need a bandwidth of only few kilobits per second or round-trip delays of seconds, while others require gigabits per second with only minimal delay.

Faced with this broad spectrum of requirements, it seems that any single network technology will be insufficient. Consequently, different notions of hybrid architectures were identified as promising approaches. In their March 2001 guest editorial for IEEE Communications Magazine, Alan Hill and Fabio Neri formulated this insight into optical networks:

"Furthermore, from an overall network perspective, a hybrid solution combining the merits of fast (optical) circuit switching with those of optical packet switching may offer better cost and performance. Indeed, such solutions may reduce the throughput requirements of packet switches."

Extending hybrid architectures even further to the complete spectrum of optics and electronics, we believe that network designers should optimally combine the strengths of both domains and at the same time diminish their weaknesses. Only recently, Vincent Chan worded it even more strongly in his November 2004 editorial for IEEE JSAC: 


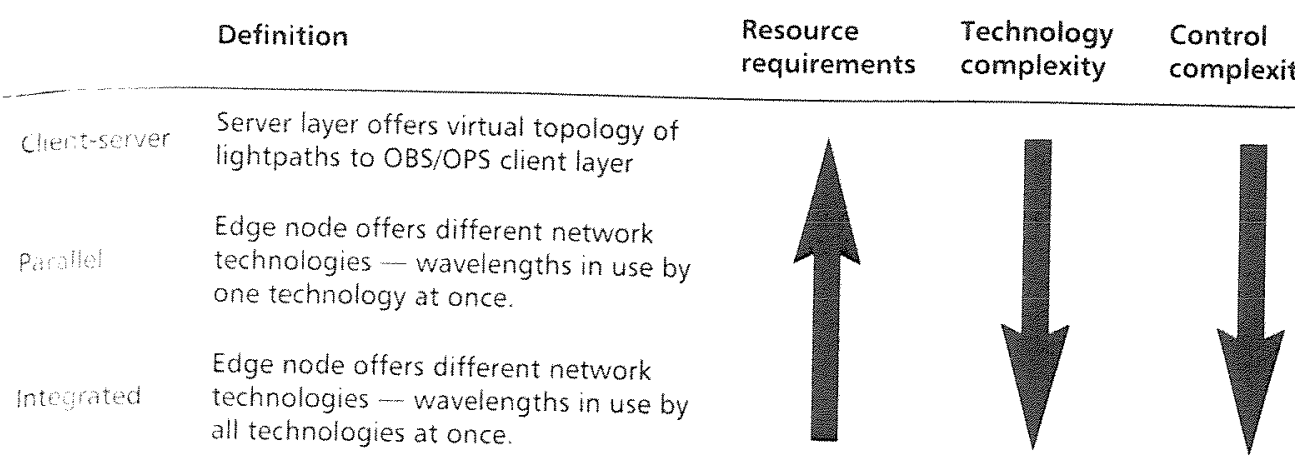

Table 1. Definition of the classes of hybrid oprical networks and their findamental chanacteristics.

- Methotion is that the ultimate solutions Whe be alloptical as the purists would have it, ma has hybrials that make ase of he best of whit and electronics. Moore is Law and traditionWherentata network is the proverbial glass with that has to be broken. I urge all of you to wetrar bour imagination and have fun at it."

L con before Vincent Chan urged us, some rescirchers had already had some (initial) fun coming up with hybrid architectures. In this article we facus on hybrid network architectures: architectures that do not apply one network technology to trinsport all traffic, but instead combine several whituing technologies into one architecture.

While a lot of ingenious ideas in that sense hat been proposed recently in major IEEE publications, this field is not structured yet. Thu we introduce a first classification and illusthat the strengths and typical characteristics of hyldit optical networks by zooming in on two specilic representatives. We also provide some pertimance metrics to give a feeling for what hen its each way of working may bring.

\section{CLASSIFICATION OF HYBRID OPTICAL NETWORK ARCHITECTURES}

For this article, in order to avoid misinterpretations wh ther hybrid concepts in the area of optical now onks, we define the term hybid as follows: lin optical network architectare is called what if it cumbines two or more basic network "wologies at the same time."

th the context of optical networks, network techologies refer to packet [1] and burst switching s well as wavelength. Waveband, and fiber hing [3]. We decided to focus on architectures bining packethurst and wavelength switching. Aly beause their combination is particulary actue: they define cotirely different requireGor the optical nemork. While packet and whehing for instance. require burst-modeHe trancelens and transmission links with

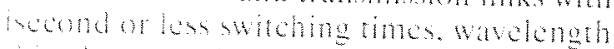
ching has more herequmbents (c.g. reconwhin the of second and higher).

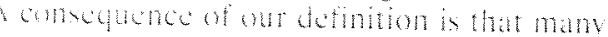
ork wemologies. He opteal burs whehing

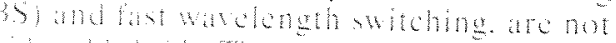

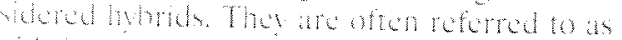

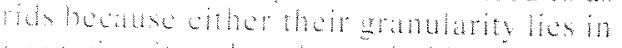

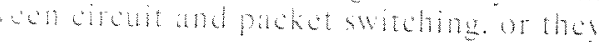

combine electronic control and optical switching. However, they are not covered by our definition mandating multiple network technologies to be deployed simultaneously.

Existing proposals for hybrid optical networks covered by the above definition can be divided into three classes, based on the degree of interaction and integration of the network technologies:

- Client-server

- Parallel

- Integrated

Next, we define these classes in progressing levels of integration and show where current proposals for architectures belong. Later, this classification and major characteristics are summarized in Table 1.

\section{Client-Server Hybrid Optical Networks}

The first class employs a hierarchy of optical layer networks with different network technologies. Adopting International Telecommunication Union - Telecommunication Standardization Sector (ITU-T) terminology, the lower of two adjacent layer networks functions as a server layer setting up a virtual topology for the upper client layer. For efficient network design, a hierarchy of bandwidth granularities is established with finer granularities in the upper layers and coarser granularities in the lower layers.

This client-server principle is not only commonly applied in electro-optical transport network architectures today (e.g., in IP-WDM or IP-SDHWDM). It is also proposed for the optical layer with respect to wavelength channels, wavebands, and fibers [3], and supported by generalized multiprotocol label switching (GMPLS) control planes. In this article, however, we focus on architectures in which the client layer is an OBS or optical packet switching (OPS) network, and the server layer is a wavelength switching netwerk.

Figure I shows such a hybrid network [1, 4. 5). OBS or OPS nodes mostly aggregate traffic at the edge of the core newwrt. These nodes are interconnected across the che network by direet lightpaths in the underlying wavelengthswitched network. Optical bursts packets aro only switehed in the client layer moles and transparenty flow in lightpaths through the circuit switched sorver hyer nodes. If the chenther nodes do mot wheh transit maffe [1. 4]. We term

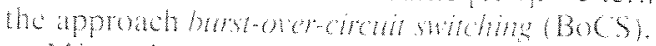

Migraton scenarios and partoulary the

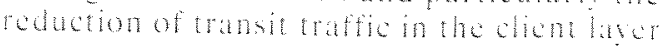

An optical network

architecture is called

hybrid if it combines

two or more basic

network

technologies at the

same time. In the

context of optical

networking, network

technologies refer to

packet and burst

switching as well as

wavelength,

waveband, and fiber

switching. 


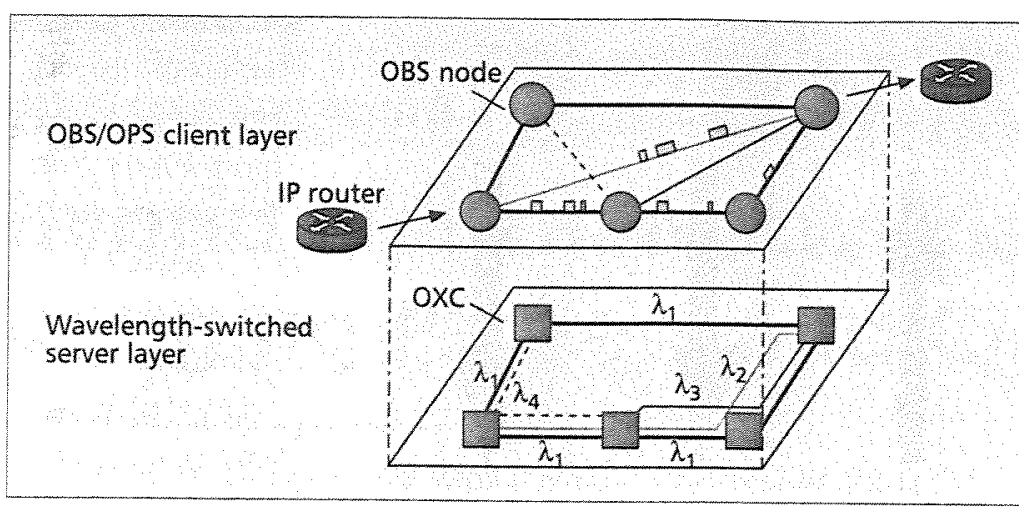

Figure 1. Client-server hybrid optical networks.

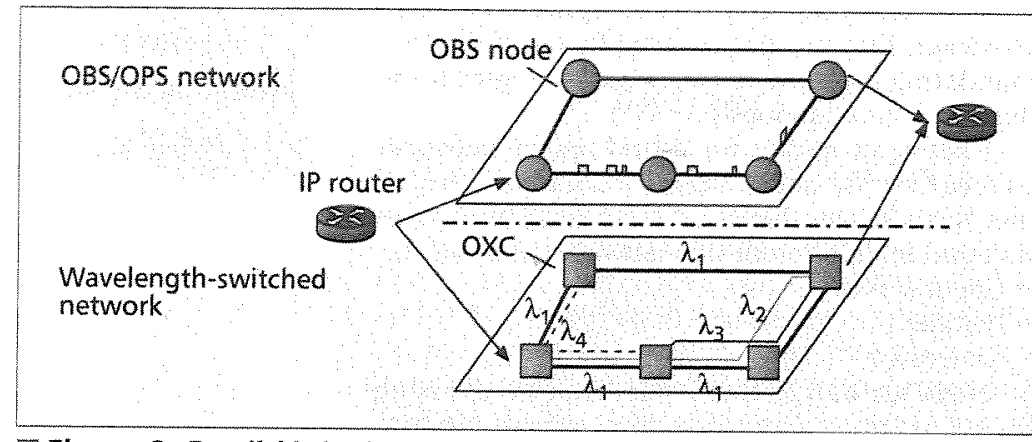

Figure 2. Parallel hybrid optical networks.

nodes clearly motivate such virtual topologies for OBS and OPS. Offloading traffic to direct lightpaths and bypassing intermediate OBS and OPS nodes reduces the number of burst-mode-capable switch interfaces in the network. Also, it avoids control processing and contention situations [6]. In addition, capacity adaptation and failure recovery benefit from dynamic lightpath capabilities in the server layer. However, most research on OBS/OPS so far has concentrated on single-layer scenarios, assuming a given physical topology in order to study fundamentals first.

Due to the lack of flexible memory in the optical layer, OBS and OPS depend on a high statistical multiplexing gain on network links to achieve a high utilization for the target quality of service (QOS). As increasing the connectivity of a network by virtual topology links yields less traffic per link and thus reduced multiplexing gain, only lower utilization can be achieved. Consequently, a dense virtual topology saves resources in bypassed intermediate nodes but requires additional resources at end nodes to compensate for this reduced utilization. Thus, the virtual topology has to be carefully designed to reduce overall network cost. Note that this effect also applies to synchronous digital hierarchy/synchronous optical network (SDH/SONET) or WDM virtual topologies. However, it is less pronounced there because extensive buffering is avalable in the IP routers of the client layer.

As noted above, OBS and OPS mandate a burst-mode-capable transmission infrastructure and often apply orthogonal modulation schemes for control information, such as frequency shift keying (FSK). Hence, the underlying wavelength-swiched transmission infrastructure also has to be burst-mode-capable and transparent to modulation schemes used in the client layer.

Client and server layer networks can be under the control of more than one operator. In that case, the client layer operator leases wavelength services to set up virtual topology links. Network control is free to apply an overlay model with separate state information, an augmented model with a higher degree of information sharing, or a peer model with complete information sharing.

\section{PARAllel HYbrid OPTICAL NeTwORKS}

In the second class of hybrid optical networks two or more optical layer networks, offering different transport services, are installed in parallel. An intelligent edge node employs them individually or in combination to optimally serve customer service requirements.

Virtual optical networks (VONs) [2] and polymorphic multiservice optical networks (PMONs) [7] introduce frameworks and possible realizations of this class of hybrid networks. They combine wavelength-switched transport (from permanent to switched) and highly dynamic OBS. Figure 2 illustrates this class with the service edge node selecting the transport service from the two networks. Here, IP traffic can be either transported in optical bursts or as a continuous byte stream inside a lightpath. In the realizations in $[8,9]$ edge nodes select a network technology based on explicit user request, traffic characteristics like bandwidth and expected flow duration, or QoS requirements.

Resources for transmission and switching can be either dedicated to or shared among the different network technologies. In both cases the assignment of network resources to the respective technology can be static or dynamic. Note that even in the case of dynamic assignment the timescale of "handover" of resources is at least on the order of wavelength path setup/teardown. This is in contrast to the last class discussed below, which supports sharing on a per packet basis.

While sharing of resources in general improves resource utilization, it also mandates that transmission and switching equipment satisfy the requirements of the most demanding technology. For instance, an integrated switch for hybrid OBS and wavelength switching [7-9] requires the faster switching technology, more sophisticated control logic, and burst-mode transceivers of OBS. Instead, all wavelength-switched services could be implemented with less complex and thus more cost-efficient technology. Similarly, sharing of fiber infrastructure requires burst-mode-capable transmission equipment. Consequently, the design of such parallel hybrid architectures has to tradeoff efficiency and realization complexity.

Similar to the arguments on resource sharing, a unified control plane across all optical layer networks facilitates network operation [7] but also requires that all specifics of the different network technologies were considered in its design and implementation. This increased complexity could slow down standardization, product development, and consequently deployment.

Note that these trade-offs regarding resource sharing and realization complexity as well as the degree of integration of the control planes apply even more to the class of integrated hybrid optical networks, discussed below. 
So far, we have implicitly assumed that the core network not only offers multiple transport services but also makes the transport decision. However, the customer could also decide on the transport service to we. This is basically proposed and implemented in CHEETAH [10]. There, a high-capacity customer has two network attachments, and chooses between a primary circuit-switched service (SDH) and a secondary TCP/IP-based transport service that is used if the primary is blocked.

\section{INTEGRATEd HYBRID OptICAL NetWORKS}

White the second class takes two or more network technologies side by side instead of the more classical cliont-server approach, the final class goes even a step further: it integrates them completely. This means all network technologies share the same bandwidth resources in the same network simultaneously. In case of a combination of wavelength switching and packet/burst switching, this means that traffic is either transported in wavelengthswitched or burst-switched mode. Each node can: - Opt to use a given wavelength segment as part of a (predetermined) wavelength path and send traffic wavelength-switched

- Ignore the established wavelength path and have a neighbor node process the traffic, even if in wavelength-switched regime it should have bypassed this neighbor

Switching between the two views (i.e., seeing the network as a collection of end-to-end lightpaths or as point-to-point wavelength segments) is done on the fly and possibly on a per packet basis.

Consider Fig. 3. Each node comprises a wavelength-switched device and a packet-switched one. Usually, a node will transmit packets over the end-to-end lightpath, since it removes the need for intermediate processing by subsequent nodes. However, in case of congestion it can change to packet-switched mode. Alternatively, the choice between the two modes can also be motivated by QoS differentiation (e.g., wavelength-switched for high-priority traffic).

This method is - theoretically - optimal from a resource viewpoint. For well behaved smooth traffic, wavelength paths can be used, while dynamic traffic can be handled by employing the packet-switched mode. However, it is also the most complex, from both technology and control points of view. As each node sees the entire network in two ways, a full integration of the wavelength-switched and the packetswitched data and control plane is needed.

To the best of our knowledge, currently only two proposals fall into this category: OpMiGua [11) and Overspill Routing in Optical Networks (ORION) [12]. In both architectures each node has to be able to detect the current mode of operation of each packet, and insert and remove packets withut disturbing existing traffic in wavelengths. Awiding collisions is far from trivial, and both appratehes rely on advanced optical components.

In the OpMiGua concept, sending nodes use two urthogonal polarization states to mark the two tramsort modes. At the next node a polarization splinter directs the packet to the responsble section of the node (i.e., an OXC or electronic packet switely. The main challenge lies in the physical rathation, for example, making stre the signal is mater and polatiation state is conecty maintained.

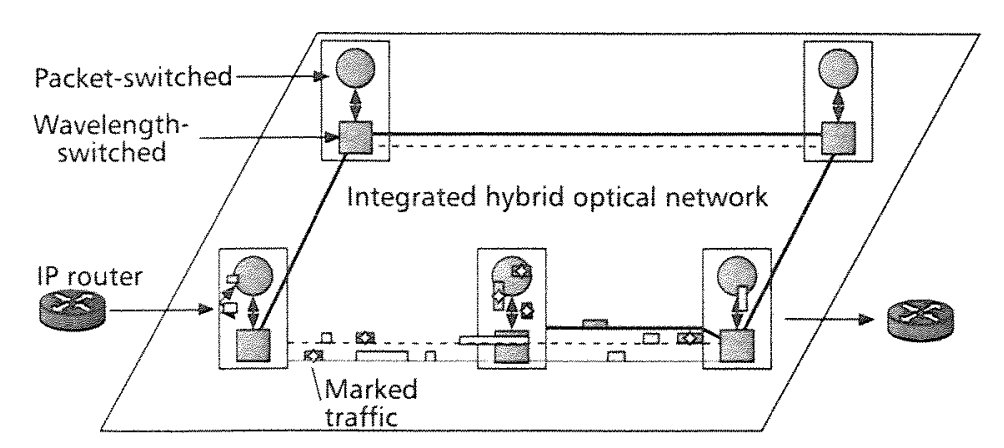

Figure 3. Integrated hybrid optical networks.

ORION considers several options, which can be divided in two broad categories. One uses a lower-bit-rate signal with an orthogonal modulation format, while the other relies on advanced all-optical detection.

Note that for both integrated hybrid architectures there must be a possibility to share resources in the first place, which de facto means to support packet switching with a possibility of inserting/removing packets into lightpaths ad hoc. This in turn means either elaborate clock (re)synchronization techniques and/or burst-mode transceivers, at least for the packet-switched mode. While the network side and logical operation of these two types seem to be sound, clearly the physical implications are very challenging if aiming for deployment in the near term.

\section{Detalled DisCusSion OF KEY HYBRID ARCHITECTURES}

After our general classification, we present two specific architectures in more detail to further illustrate the characteristics of hybrid architectures. First, the optical burst transport network (OBTN) architecture [5] is introduced as an example of a client-server hybrid optical network. Second, ORION is discussed as an example of an integrated hybrid optical network, in context of an electronic packet router integrated with an $\mathrm{OXC}$. While this discussion only covers the case of a static wavelength-switched layer, it can be extended to the dynamic case directly. Dimensioning studies analyze both architectures using the pan-European reference core network [12] with 16 nodes, 23 fiber links, and the traffic demand matrix for 2008.

\section{OPTICAL BURST TRANSPORT NETWORK}

This section outlines the OBTN architecture, which offers more resource- and cost-efficient transport of optical burst data than OBS. OBTN combines a dense virtual topology and constrained alternative routing with effective contention resolution strategies in order to reduce the number of ports in burst-switched nodes, while maintaining a high network efficiency. It targets the transport of optical bursts originating in optical feeder networks (e.g. MANs) across the core network [5].

First, OBTN applies a dense virtual topology in which nodes are connected by direct lightpaths optically bypassing intermediate nodes. The interconncetion pattern is defined by tranto 

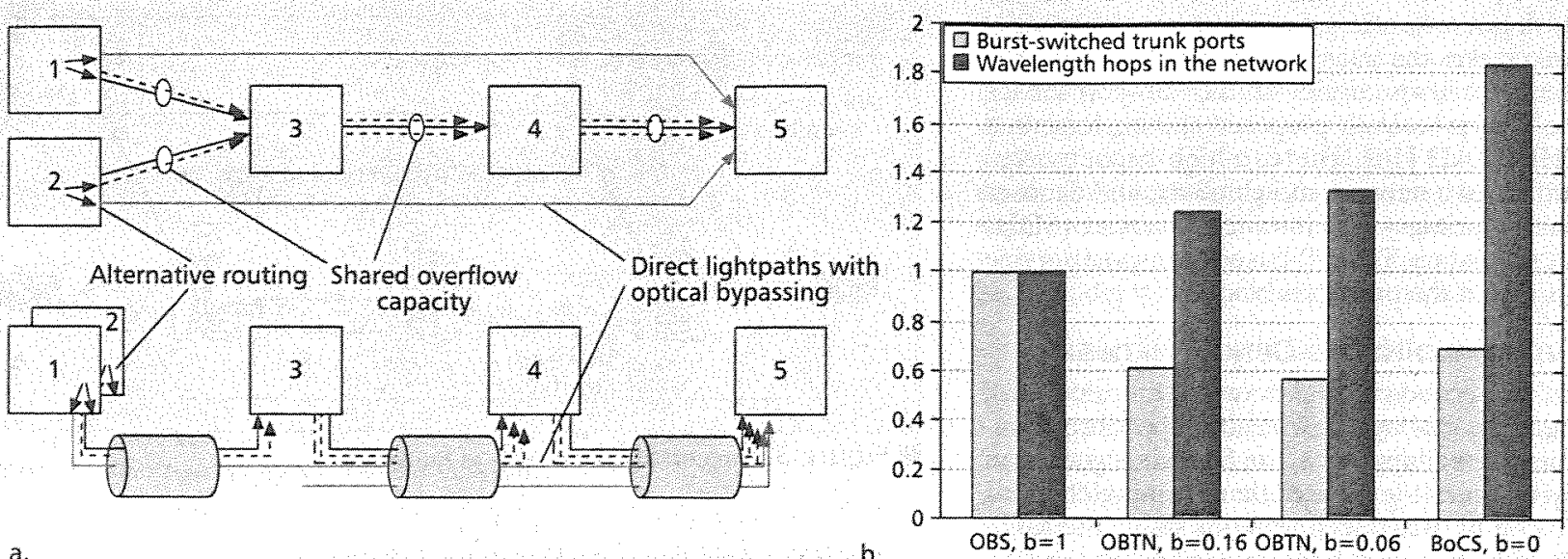

a.

b.

Figure 4, a) OBTN network and node view for sample network with five nodes, in which direct lightpaths (solid) are set up for the assumed traffic demands, and shared overflow capacity (dashed) is allocated for alternative routes of relations 1-5 and 2-5; b) comparison of node and network resources for $O B S, O B T N$, and BoCS.

demands or operational criteria. Second, as statistical multiplexing on a large number of network links each with small capacity can be inefficient, OBTN has two additional provisions also illustrated in Fig. $4 \mathrm{a}$ :

- Bursts use constrained alternative routing in case of contention on direct lightpaths.

- A small amount of shared overflow capacity is allocated to links used by alternative paths.

In order to achieve high network resource efficiency despite reduced statistical multiplexing gain per virtual link, both wavelength conversion and a very simple shared FDL buffer are used.

In OBS, unconstrained alternative routing commonly referred to as deflection routing - can lead to high delay variation, thus burst reordering, and potentially negative effects on TCP. OBTN avoids this by constraining alternative routes to virtual links, which follow the same fiber links in the physical topology as the primary route. Thus, all bursts with the same OBTN ingress and egress nodes experience the same propagation delay, the dominating delay component in WANs.

Introducing alternative routing shifts some traffic from virtual links, which were dimensioned for this traffic, to other virtual links, which were not. This yields an undesired mismatch of routing and dimensioning and thus performance degradation. To circumvent this, some extra capacity, termed shared overflow capacity, is allocated in the network for traffic on alternative routes. In order for this capacity to be shared by many alternative routes, it is allocated to virtual links connecting neighbor nodes in the physical topology.

OBTN can be characterized by the share of network capacity, $b, 0 \leq b \leq 1$, allocated as shared overflow capacity. This notation also comprises the OBS architecture $(b=1$, virtual and physical topology are identical, all capacity is shated) and the BoCS architectures presented in $[1,4](b=$ 0 , full-mesh virtual topology, only dedicated capacity), which allows the following unified arehitecture comparison.

Figure $4 \mathrm{~b}$ quantifies the dimensioning of node and network resources for OBTN, OBS, and
BoCS. For OBTN two different dimensioning values of shared overflow capacity are shown $(b$ $=0.06$ and 0.16 ). The number of trunk interfaces in burst-switched nodes and the number of wave length hops in the physical fiber infrastructure describe the node and network resources, respectively. The comparison is performed for a burst loss probability in the network of $10^{-4}$. All values shown are relative to those of OBS.

On the one hand, BoCS and especially the OBTN architecture successfully economize on the number of expensive burst-switched ports while OBS requires the highest number here due to the high transit traffic. On the other hand, both OBTN architectures only have a limited increase in wavelength hops compared to OBS, while BoCS is severely penalized due to its low network efficiency.

Applying the cost relations for optical networks, in which network resources are increasingly considered commodities and node resources remain the major cost driver, OBTN constitutes an effective solution to reduce overall cost. Tuning the ratio of shared overflow capacity $b$ to account for a specific cost relation of burst-switched ports and wavelength hops yields optimal OBTN architectures.

\section{OVERSPILL ROUTING IN OPTICAL NETWORKS}

In this section we describe a particular instance of an integrated hybrid optical network, ORION. As mentioned, ORION employs markers to be able to instantly change between wavelengthswitched and packet-switched operation mode on the same wavelength. Here, we assume such a marker exists by way of a low-bit-rate frequency shift keying (FSK) signal accompanying an intensity modulated payload. The packet-switched mode is called overspill mode, while the wavelength-switched mode is called nomal mode.

Figure 5 a shows a complete example ORION node. The OXC (1) has four wavelengths com ing in, of which two, $\lambda_{2}$ and $\lambda_{3}$, are terminated toward the electrical IP router (2). The two others, $\lambda_{1}$ and $\lambda_{4}$, pass through transparently (3). To detect the marker, we split off some of the power at ove are

is $\mathrm{p}$

so 1 fort inte not suf con pac rec OR eith orig mo rem nee: The idle pacl 

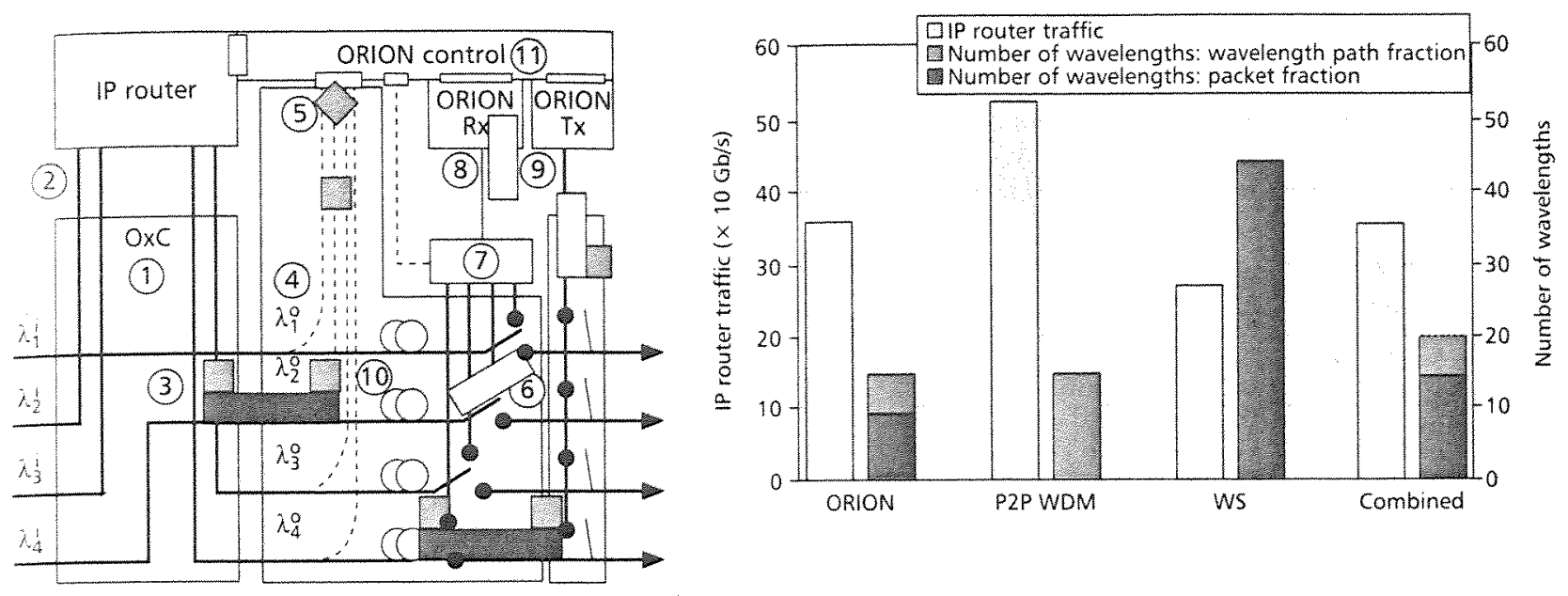

b.

Figure 5. a) An example design of an ORION node; b) results of a dimensioning case study.

at $(4)$ and process it at (5). If it indicates an overspill packet, $1 \times 2$ fast optical switches $(6)$ are set up toward the electrical IP router. Traffic is preferably sent in wavelength-switched mode, so we expect very few overspill packets. Therefore, drop wavelengths are combined (7) to save interface cards. Likewise, $1 \times 2$ switches need not be installed on all wavelengths; a subset may suffice. In the rare case of contention at the combiner, a packet drop cannot be avoided.

After the $1 \times 2$ switch is set, the overspill packet can be received through a wideband receiver (8). The added simple electronic ORION control block can then decide (11) to either send it via normal mode on a lightpath originating at that IP router, or again in overspill mode (9). In standard ORION a packet always remains in overspill mode (see [12] for variants).

Short delay lines (10) compensate for the time needed to read, detect, and set the $1 \times 2$ switches. The delay lines also allow detecting large enough idle periods on the wavelengths in order to insert packets in overspill mode on pass-through wavelengths by opening a window to the future. Information about this availability is sent to the ORION control module (11), the function of which now is also to control the ORION transmitter module (9). Again, to avoid interface cards on all wavelengths while keeping full flexibility, one or several tunable lasers could be installed.

The advantages of ORION are already partly reflected in the node design. First, the logic controlling the overspill part needs to work only with the markers. Second, packets that are inserted on a direct wavelength path are left untouched until they rach their destination. Thus, no expensive incrace cards at line rate are needed for passthrough traffic, delay is minimal, and delivery is onsured. Finally, since we only rely on informathon obtaned via the markers, this part of the note is totally independent of the underlying line encoling or data rate. Note that altematively, an OBSOPS switch can also replace the IP router (2) The advanages of ORION still apply: smaller OPSOBS witches (less trafte seen, less procusme) and high utilization rate.
The study in Fig. 5b compares standard ORION with three other network technologies: purely packet-switched with point-to-point WDM, purely wavelength-switched with end-toend WDM, and a parallel hybrid optical network termed combined, which operates like CHEETAH. We further assume $10 \mathrm{~Gb} / \mathrm{s}$ per wavelength installed, where each wavelength can be installed unidirectionally and independent of others.

Again, we quantify the approaches by a double metric: on one hand the amount of traffic the IP routers will have to handle, and on the other hand the amount of wavelength hops needed in the network.

When looking at average IP router load, it is very clear that point-to-point WDM has to handle a lot more traffic than the other three alternatives. The wavelength-switched WDM solution naturally performs best, but more interesting, the combined technology and ORION are close to this optimum.

When looking at average number of wavelength hops needed in the network we see the opposite. The wavelength-switched WDM architecture uses many more wavelength hops, while ORION uses the same amount of wavelength hops as the packet-switched case. We also see an important feature of ORION illustrated: only a small fraction of the wavelengths use overspill (gray area). In fact, on average, only around 1 percent of traffic was in overspill. This performance evidently comes at a cost: a bigger IP router has to be provisioned to handle overspill, but its calculated size is only marginally greater than the wavelength-switched solution. Compared to ORION, the combined approach uses slightly more wavelength hops. Another observation, not shown, is the dramatic reduction of packetswitched traffic at nodes with high amounts of transit traffic, due to ORION relying on wavelength switching as a primary transport method.

\section{CONCLUSION}

Hybrid optical network architectures that combine two or more basic network technologies at the same time open several new degrees of free- 


\section{hybrid architectures \\ define an interesting \\ route to satisfy the \\ heterogeneous \\ network require- \\ ments of the future. \\ Athough several \\ hybrid optical \\ architectures already \\ were proposed in \\ recent years, the \\ complete design \\ space is far from \\ exhaustively \\ researched.}

dom to bring packets and circuits closer together optimizing the overall network design. In order to structure this research direction, we have classified hybrid optical network architectures proposed so far based on the degree of interaction and integration. We have also discussed their key characteristics, performance benefits, and realization complexity.

As a summary, we provide short definitions of the client-sever, parallel, and integrated hybrid architecture classes in Table 1. It illustrates principal relations regarding resource requirements, technology and control complexity. While the first can be expected to increase from integrated via parallel to client-server, the opposite applies for the latter two criteria.

In a second part, we present two hybrid architectures in greater detail, one client-server and one integrated representative. In a dimensioning study for a pan-European reference network scenario, both architectures show their benefits over non-hybrid altematives.

As we have shown, hybrid architectures define an interesting route to satisfy the heterogeneous network requirements of the future. Although several hybrid optical architectures have already been proposed in recent years, the complete design space is far from exhaustively researched.

Concluding, people followed Vincent Chan's editorial advice in $J S A C$ and had fun in their research, so there is no shortage of new ideas!

\section{ACKNOWLEDGMENTS}

We would like to gratefully acknowledge Biswanath Mukherjee for invaluable discussions. This work was partly funded by the European Commission through IST-NOBEL (FP6 507509) and NoE E-Photon/ONe. The Flemish government also funded through the IWT-GBOU project Optical Networking and Node Architectures, and through a Ph.D. grant to E. Van Breusegem.

\section{REFERENCES}

[1] M. 1. O'Mahony et al., "The Application of Optical Packet Switching in Future Communication Networks," IEEE Commun. Mag. vol. 39, no. 3, 2001

[2] C. Qiao and M. Yoo, "Optical Burst Switching (OBS) -. A New Paradigm for an Optical Internet, "J. High Speed Networks, vol. 8 , no. 1, 1999

3) R. Izmailov et al., "Hybrid Hierarchical Optical Networks," IEEE Commun. Mag., vol. 40, no. 11, 2002.

[4] C. T. Chou et al. "A Hybrid Optical Network Architecture Consisting of Optical Cross-Connects and Optical Burst Switches," IEEE Int'l. Conf. Comp. Commun. and Networks, Dallas, TX, Oct. 2003

[5] C. M. Gauger and B. Mukherjee, "Optical Burst Transport Network (OBTN) - A Novel Architecture for Efficient Transport of Optical Burst Data Over Lambda Grids," IEEE High Perf. Switching and Routing, Hong Kong. May 2005.

[6] $B$. Chen and J. Wang, "Hybrid Switching and p-Routing for Optical Burst Switching Networks; "IEEE JSAC, vol. 21, no. 7,2003

171. de Miguel et al "Polymorphic Architectures for Optical Networks and Their Seamless Evolution Towards Next Generation Networks," Photonic Network Commun, vol. 8, no. 2, Sept. 2004

(3) $G$. W Lee et al. "Performance Evaluation of an optical Hybrid Switching System," IEEE GLOBECOM, San Fran cisco, CA, Dec. 2003

[9] C. Xin et al. "A Hybrid Optical Switching Approach. IEEE GLOBECOM San FranciscolCA, Dec 2003

(10) $X$. Zheng et al. "CHEETAH: Circult-switched High-Speed End-to-End Transport Archtecture Testbed" "IEEE Commun Mag. Opt. Conmun. Supp, vot 3, no. 3, 2005
[11] 5. Bjornstad, M. Nord, and D. R. Hjelme "QoS Differentiation and Header/Payload Separation in Optical Packet Switching Using Polarization Multiplexing, "ProC. ECOC 2003, Rimini (Italy), Sept. 2003, Mo3.4.6, pp. 28, 29.

[12] E. Van Breusegem et al., "A Broad View on Overspill Routing in Optical Networks: A Real Synthesis of Packet and Circuit Switching?" Optical Switching and Net, vol: 1 , no. 1,2004 , pp. 51-64.

\section{BIOGRAPHIES}

CHRISTOPH GAUGER [M] (gauger@ikr.uni-stuttgart.de) received his M.S. in computer science from Georgia Tech in 1999 and his Dipl. Ing. in electrical engineering from the University of Stuttgart in 2000. Since then he has been with the Institute of Communication Networks and Computer Engineering (IKR) at the University of Stuttgart, where he leads the research group on high-speed networks. In 2004 he worked with Biswanath Mukherjee at the University of California at Davis for three months. He has been engaged in and led several research projects on architectures and traffic engineering for IP over WDM. He serves on the editorial board of Optical switching and Networking and is a member of VDE ITG and the Network of Excellence E-Photon/ONe

ERIK VAN BREUSEGEM [M] (erik.vanbreusegem@intec.ugent. be) received his $M . S c$. degree in computer science and his Ph.D. degree from Ghent University in 2001 and 2006 . respectively. He was working at the same university as researcher in the Department of Information Technology (INTEC) as part of the research group INTEC Broadband Communication Networks (IBCN) headed by Prof. Piet Demeester. His research deals with optical networks in general, with a focus on hybrid optical network designs. He was involved in this context in several IST projects (STOLAS, NOBEL, LASAGNE) and in COST-Action 266. His work has been published in more than 30 scientific publications in international conferences and journals.

PAuL J. KüHN [F] (kuehn@ikr.uni-stuttgart.de) received Dipl.Ing. and Dr.-Ing. degrees in electrical engineering from the University of Stuttgart, Germany, in 1967 and 1972 . respectively. In 1977 he joined Bell Laboratories. Holmdel New Jersey, where he worked in the field of computer communications. Since 1982 he has held the chair of Com munication Networks and Computer Engineering at the University of Stuttgart. His areas of interest are communication network architectures and protocols, computer engineering, and performance modeling and evaluation. He is a member of ACM, ITG, GI, and various academies of sciences. He is Chairman of the International Advisory Council IAC of the International Teletraffic Congress (ITC).

MARIO PICKAVET [M] (mario.pickavet@intec.ugent.be) received $M . S C$. and $P h . D$. degrees in electrical engineering specialized in telecommunications, from Ghent University in 1996 and 1999 , respectively. Since 2000 he is professor at Ghent University where he teaches courses on multimedia networks and network modeling. His current research interests are related to broadband communication networks (WDM, IP, (G-)MPLS, OPS, OBS), and include design, long-term planning, and routing of core and access net works. In this context he is currently involved in the European IST projects LASAGNE and e-Photon/ONe. He has published over 150 international publications in both jour nals and proceedings of conferences. $\mathrm{He}$ is one of the authors of the book Network Recovery: Protection and Restoration of Optical, SONET-SDH, IP, and MPLS

PIET DEMEESTER [SM] (Piet.Demeester@intec ugent be) received his M.Sc. degree in electrical engineering and his Ph.D. degree from Ghent University in 1984 and 1988 respectively. In 1992 he started a new research activity on broadband communication networks resuling in the INTEC Broadband Communications Network (BCN) research group. In 1993 he became a protessor a Ghent University. where he is responsible for research and education on communication networks. His research activities cover various (IP, ATM, SDH, WDM, access, active, mobile) communi cation networks, and include network planning, network and service management, telecom sofware, internetwork ing. network protocols for QoS support, and so torth. He is an author of more than 400 publications in the area of network design, optimization, and management. He is a member of the editonial board of several hitemational jour nals and has been a nember of several technical program commitees $\angle E C O C$ OFC, ORCN, 1CCCN, and 125 : 


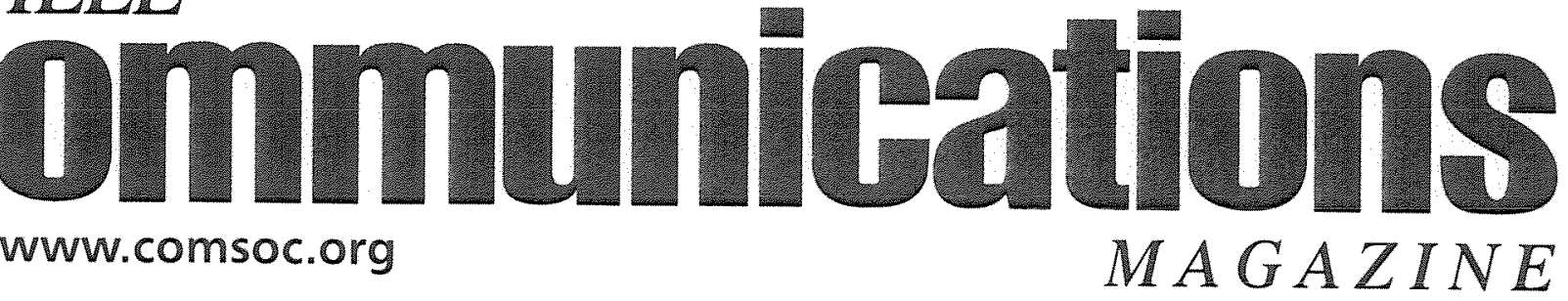

\section{Optical Communications}

- Overview of the Optical Broadband Access Evolution

- QoS in Optical Packet-Switched Networks

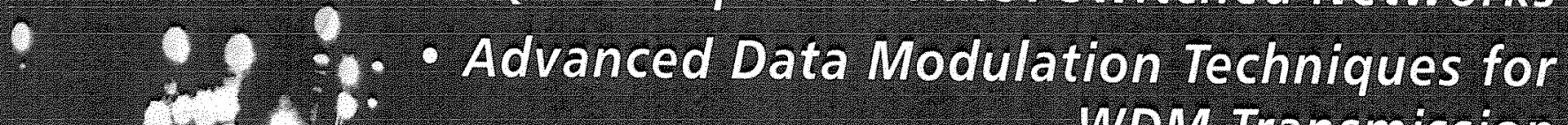
r $\because\}^{2}$ WDM Transmission

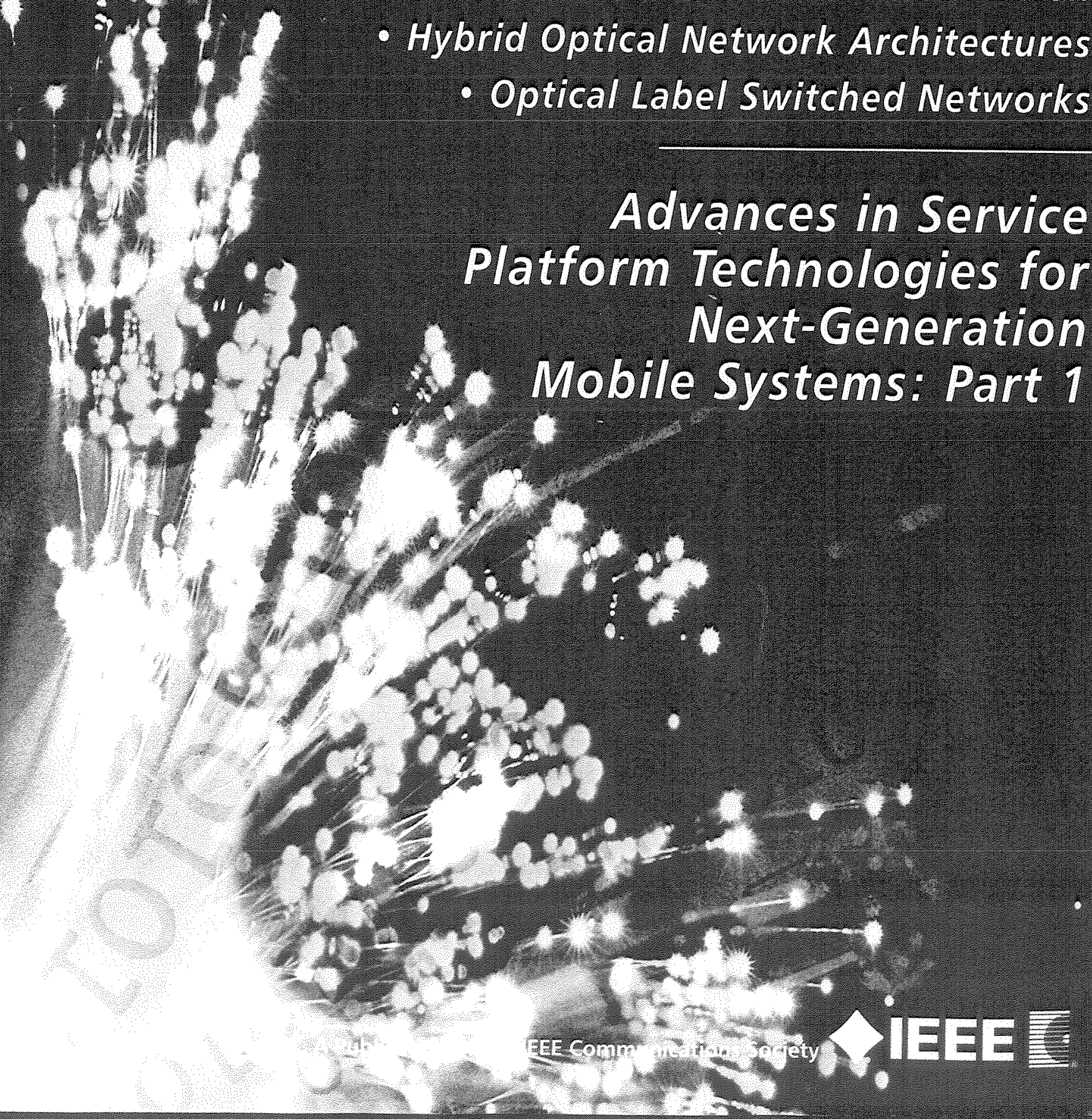

